\title{
Modified Differential Transform Method for Two Singular Boundary Values Problems
}

\author{
Yinwei Lin, Hsiang-Wen Tang, and Cha'o-Kuang Chen \\ Department of Mechanical Engineering, National Cheng Kung University, Tainan 70101, Taiwan \\ Correspondence should be addressed to Cha'o-Kuang Chen; ckchen@mail.ncku.edu.tw
}

Received 6 September 2013; Accepted 17 November 2013; Published 5 January 2014

Academic Editor: Ying $\mathrm{Hu}$

Copyright ( 2014 Yinwei Lin et al. This is an open access article distributed under the Creative Commons Attribution License, which permits unrestricted use, distribution, and reproduction in any medium, provided the original work is properly cited.

\begin{abstract}
This paper deals with the two singular boundary values problems of second order. Two singular points are both boundary values points of the differential equation. The numerical solutions are developed by modified differential transform method (DTM) for expanded point. Linear and nonlinear models are solved by this method to get more reliable and efficient numerical results. It can also solve ordinary differential equations where the traditional one fails. Besides, we give the convergence of this new method.
\end{abstract}

\section{Introduction}

In the present paper, we consider the following two singular boundary value problems (BVPs) of second order:

$$
\begin{aligned}
& u^{\prime \prime}(t)+\frac{f(t)}{t(t-1)} u^{\prime}(t)+\frac{g(t)}{t(t-1)} N(u(t)) \\
& =\frac{h(t)}{t(t-1)}, \quad 0<t<1, u(0)=p, u(1)=q,
\end{aligned}
$$

where $f(t), g(t)$, and $h(t)$ are known and continuous functions, $t \in(0,1)$, and $N(u)$ is a nonlinear function of $u$. The equation is singular at these two boundary values $t=0,1$.

Scientists and engineers have been interested in the singular equation because of its importance in many applications such as physical and mathematical models. There are many research directions on these equations. Some studied their qualitative properties [1, 2]. For example, Bartolucci and Montefusco [1] studied the concentration-compactness problem and the mass quantization properties. Some others used theorems to establish the existence and uniqueness of solution [3, 4]. For example, Guo et al. [3] got the existence and uniqueness of solution using a fixed point theorem.

Recently great attention had been paid to numerical solutions [5-13]. For example, Duan and Rach [5] solved boundary value problems using a new modified Adomian decomposition method. Chowdhury and Hashim [7] used homotopy asymptotic method for finding the approximate solutions. Wazwaz $[6,8,9]$ used Adomian decomposition method to get the numerical solutions.

Puhov in 1976 [14] proposed the concept of DTM. DTM is the extension of Taylor series method and had been applied to solve analytic solutions of ordinary $[15,16]$, partial [17-19], differential-algebraic equations [20, 21], differentialdifference equations [22, 23], and integrodifferential equations [24, 25]. Numerical solutions are also obtained [26]. Furthermore, Alquran and Al-Khaled [27] applied DTM to solve some eigenvalue problems.

In the present paper, there are two singular points and these two singular points are just boundary values of the equation. Traditional DTM only can solve one-point singular BVP or two-point BVP (but not both the two points are just boundary values). We add some operation properties of DTM; then DTM can be used to calculate this type of problem. Furthermore, we have a convergent analysis of this method.

\section{Modified Technique}

Now, we have a brief description of standard DTM.

Let $u(t)$ be an analytic function in a domain $D$ and let $t=a$ represent any point in $D$. The function $u(t)$ is then 
represented by a power series whose center is located at $a$. Then Taylor series expansion of $u(t)$ is expressed as

$$
u(t)=\sum_{k=0}^{\infty} \frac{(t-a)^{k}}{k !}\left[\frac{d^{k} u(t)}{d^{k}}\right]_{t=a}, \quad t \in D .
$$

The particular case of (2) when $a=0$ is referred to as the Maclaurin series expressed as

$$
u(t)=\sum_{k=0}^{\infty} \frac{t^{k}}{k !}\left[\frac{d^{k} u(t)}{d^{k}}\right]_{t=0}, \quad t \in D .
$$

The differential transformed function $u(t)$ is defined as

$$
U(k) \equiv \frac{H^{k}}{k !}\left[\frac{d^{k}(t)}{d t^{k}}\right]_{t=0}, \quad k=0,1,2, \ldots,
$$

where $U(k)$ represents the transformed function and $u(t)$ is the original function. The differential spectrum of $U(k)$ is confined in the interval $t \in[0, H] ; H$ is the given constant number.

The differential inverse transform of $U(k)$ is defined as

$$
u(t)=\sum_{k=0}^{\infty}\left(\frac{t}{H}\right)^{k} U(k) .
$$

From the definition, the differential transformation technique is based upon the Taylor series expansion. In real applications, it is found that the number of arguments required to restore the unknown function precisely can be reduced by specifying an appropriate value of the constant $H$. In other words, the function $u(t)$ can be expressed in terms of a finite series as follows:

$$
u(t)=\sum_{k=0}^{n}\left(\frac{t}{H}\right)^{k} U(k) .
$$

Next, we state some important properties of the Taylor differential transformation derived using the expressions in (4) and (5) which are needed in the sequel.

\section{The Operation Properties of Differential Transformation}

If $U(k)$ and $V(k)$ are the transformed functions corresponding to $u(t)$ and $v(t)$, then the fundamental mathematical operations of differential transformation are listed as follows. (For 9-12 is new, the proof of the others can see references.)

(1) If $z(t)=u(t) \pm v(t)$ then $Z(k)=U(k) \pm V(k)$.

(2) If $z(t)=a u(t)$ then $Z(k)=a U(k)$.

(3) If $z(t)=d^{m} u(t) / d t^{m}$ then $Z(k)=(k+1)(k+2) \cdots(k+$ $m) U(k+m)$.

(4) If $z(t)=u(t) v(t)$ then $Z(k)=\sum_{i=0}^{k} u(i) v(k-i)$.

(5) If $z(t)=t^{m}$ then

$$
Z(k)=\delta(k-m)= \begin{cases}1, & k=m, \\ 0, & k \neq m .\end{cases}
$$

(6) If $z(t)=\exp (b t)$ then $Z(k)=b^{k} / k$ !.

(7) If $z(t)=\sin (c t+a)$ then $Z(k)=\left(c^{k} / k !\right) \sin ((\pi k / 2)+a)$.

(8) If $z(t)=\cos (c t+a)$ then $Z(k)=\left(c^{k} / k !\right) \cos ((\pi k / 2)+$ a).

Next, the following lemmas are new, so we have to prove them.

(9) If $z(t)=t^{n} d(t)$, then $Z(k)=D(k-n)$.

Proof. For simplicity, letting $H=1$

$$
\begin{aligned}
d(t) & =\sum_{k=0}^{\infty} t^{k} D(k) \\
& \Longrightarrow t^{n} d(t)=\sum_{k=0}^{\infty} t^{n+k} D(k)=\sum_{i=n}^{\infty} t^{i} D(i-n) \\
& =\sum_{k=n}^{\infty} t^{k} D(k-n) .
\end{aligned}
$$

(10) Consider

$$
z(t)=t^{2} \frac{d^{2} u(t)}{d t^{2}} \Longrightarrow Z(k)=k(k-1) U(k) .
$$

Proof. For $y(t)=d^{2} u(t) / d t^{2} \Rightarrow Y(k)=(k+2)(k+1) U(k+2)$; for $z(t)=t^{2} y(t) \Rightarrow Z(k)=Y(k-2)=k(k-1) U(k)$.

(11) One has

$$
z(t)=t \frac{d^{2} u(t)}{d t^{2}} \Longrightarrow Z(k)=(k+1) k U(k+1)
$$

Proof. Similar method as (9).

(12) One has

$$
z(t)=t \frac{d u(t)}{d t} \Longrightarrow Z(k)=k U(k)
$$

Proof. Similar method as (9).

\section{Convergence Analysis}

Theorem 1. Consider the following two singularly linear BVPs:

$$
t(1-t) u^{\prime \prime}(t)+(1-t) u^{\prime}(t)+g(t) u(t)=f(t), \quad 0<t<1,
$$

where $f(t)=f_{0}+f_{1} t+f_{2} t^{2}+\cdots$ and $g(t)=g_{0}+g_{1} t+g_{2} t^{2}+\cdots$.

If there exists a fixed $n$ such that $n \geq m,\left|f_{k}\right| \leq M r^{k}$ for some fixed $M, 0<r<1$, all $k \geq n$, and $U(n) \leq M r^{n}$, then the numerical solution using the present method absolutely converges. 
Proof. For simplicity, let $g(t)=g_{0}$. The general case is similar to the special case.

By using (9) and (10), we have

$$
\begin{gathered}
t^{2} u^{\prime \prime}(t) \Longrightarrow k(k-1) U(k) \\
t u^{\prime \prime}(t) \Longrightarrow(k+1) k U(k+1) .
\end{gathered}
$$

So the differential transformation of (12) is

$$
\begin{gathered}
(k+1) k U(k+1)-k(k-1) U(k)+(k+1) U(k+1) \\
-k U(k)+g_{0} U(k)=F(k), \quad k \geq m,
\end{gathered}
$$

for fixed $m$. So we have

$$
U(k+1)=\frac{1}{(k+1)^{2}}\left[F(k)+\left(k^{2}+g_{0}\right) U(k)\right] .
$$

Suppose $|U(k)| \leq M r^{k}$ is true for all $k \geq n \geq m$. From (15), we have

$$
\begin{aligned}
|U(k+1)| & \leq \frac{1}{(k+1)^{2}}\left[M r^{k}+\left(k^{2}+g_{0}\right) M r^{k}\right] \\
& =M r^{k}\left[\frac{1+k^{2}+\left|g_{0}\right|}{(k+1)^{2}}\right] .
\end{aligned}
$$

Let $r=\left(\left(1+k^{2}+\left|g_{0}\right|\right) /(k+1)^{2}\right)<1$ for $k$ is large enough. By induction, the hypothesis is true. So we have

$$
|u(t)| \leq \sum_{k=0}^{\infty} t^{k}|U(k)| \leq \sum_{k=0}^{\infty}|U(k)| \leq M \sum_{k=0}^{\infty} r^{k}=\frac{M}{1-r} .
$$

Next we have the theorem for nonlinear BVP.

Theorem 2. Consider the following two singularly nonlinear BVPs

$$
\begin{gathered}
t(1-t) u^{\prime \prime}(t)+(1-t) u^{\prime}(t)+g(t) u(t) \\
+u(t)^{2}=f(t), \quad 0<t<1,
\end{gathered}
$$

where $f(t)=f_{0}+f_{1} t+f_{2} t^{2}+\cdots, g(t)=g_{0}+g_{1} t+g_{2} t^{2}+\cdots$.

If there exists a fixed $n$ such that $n \geq m,\left|f_{k}\right| \leq r^{k}, 0<r<$ 1 , all $k \geq n$, and $U(n) \leq r^{n}$, then the numerical solution using the present method absolutely converges.

Proof. For simplicity, let $g(t)=g_{0}$. The general case is similar to the special case.

So the differential transformation of (18) is

$$
\begin{gathered}
(k+1) k U(k+1)-k(k-1) U(k)+(k+1) U(k+1) \\
-k U(k)+g_{0} U(k)+B(k)=F(k), \quad k \geq m,
\end{gathered}
$$

for fixed $m, B(k)=U(0) U(k)+U(1) U(k-1)+\cdots+U(k) U(0)$. So we have

$$
U(k+1)=\frac{1}{(k+1)^{2}}\left[F(k)+\left(k^{2}+g_{0}\right) U(k)-B(k)\right] .
$$

Suppose $|U(k)| \leq r^{k}$ is true for all $k \geq n \geq m$. So we have

$$
|U(i) U(k-i)| \leq r^{i} r^{k-i}=r^{k} \Longrightarrow|B(k)| \leq(k+1) r^{k} .
$$

From(20), we have

$$
\begin{aligned}
|U(k+1)| & \leq \frac{1}{(k+1)^{2}}\left[r^{k}+\left(k^{2}+g_{0}\right) r^{k}+(k+1) r^{k}\right] \\
& =r^{k}\left[\frac{1+k^{2}+\left|g_{0}\right|+k+1}{(k+1)^{2}}\right] .
\end{aligned}
$$

Let $r=\left(\left(1+k^{2}+\left|g_{0}\right|+k+1\right) /(k+1)^{2}\right)<1$ for $k$ is large enough. By induction, the hypothesis is true. So we have

$$
|u(t)| \leq \sum_{k=0}^{\infty} t^{k}|U(k)| \leq \sum_{k=0}^{\infty}|U(k)| \leq \sum_{k=0}^{\infty} r^{k}=\frac{1}{1-r} .
$$

\section{Numerical Examples}

Differential transformation method (DTM) is used to solve the following examples. Some numerical results are also compared with RKHSM in [28]. The algorithm is performed by software with 16-digit precision.

Example 1. Consider the following two singularly linear BVPs:

$$
t(1-t) u^{\prime \prime}(t)+(1-t) u^{\prime}(t)+u(t)=f(t), \quad 0<t<1,
$$

with boundary values $u(0)=0, u(1)=1$, and $f(t)=t(4-3 t)$. The exact solution is $u(t)=t^{2}$.

By using (9) and (10), we have

$$
\begin{aligned}
& t^{2} u^{\prime \prime}(t) \Longrightarrow k(k-1) U(k), \\
& t u^{\prime \prime}(t) \Longrightarrow(k+1) k U(k+1) .
\end{aligned}
$$

So the differential transformation of (24) is

$$
\begin{gathered}
(k+1) k U(k+1)-k(k-1) U(k)+(k+1) U(k+1) \\
-k U(k)+U(k)=-3 \delta(k-2)+4 \delta(k-1),
\end{gathered}
$$

where

$$
\delta(k-m)= \begin{cases}1, & k=m \\ 0, & k \neq m\end{cases}
$$

for $k \geq 2$. One has

$$
\begin{aligned}
& \Longrightarrow U(k+1) \\
& =\frac{1}{(k+1)^{2}}\left[\left(k^{2}-1\right) U(k)-3 \delta(k-2)+4 \delta(k-1)\right] .
\end{aligned}
$$


TABLE 1: Comparison of relative errors of the present method for Example 2.

\begin{tabular}{lccc}
\hline$x$ & True solution $u(x)$ & Geng $[28] U_{5,20}$ & Present $\mathrm{DTM}_{2}$ method \\
\hline 0.08 & 0.0064 & $1.91 E-06$ & 0 \\
0.16 & 0.0256 & $1.54 E-06$ & 0 \\
0.24 & 0.0576 & $1.58 E-06$ & 0 \\
0.32 & 0.1024 & $1.59 E-06$ & 0 \\
0.48 & 0.2304 & $1.20 E-06$ & 0 \\
0.64 & 0.4096 & $3.96 E-07$ & 0 \\
0.80 & 0.6400 & $6.07 E-08$ & 0 \\
0.96 & 0.9216 & $8.13 E-09$ & 0 \\
\hline
\end{tabular}

For the coefficient of constant and $t$, because $u(0)=0$, let $u(t)=U(1) t+U(2) t^{2}$; substituting in (24), we have

$$
\left(t-t^{2}\right) 2 U(2)+(1-t)[U(1)+2 U(2) t]+U(1) t .
$$

The coefficient of constant is

$$
U(1)=0 .
$$

The coefficient of $t$ that is $k=1$ is

$$
\begin{gathered}
2 U(2)-U(1)+2 U(2)+U(1)=4 \\
\Longrightarrow U(2)=1 .
\end{gathered}
$$

As $k=2$ in (28),

$$
U(3)=\frac{3 U(2)-3}{9}=0 .
$$

For $k=3,4,5, \ldots$,

$$
U(k+1)=0 .
$$

Then we have the numerical solution of the present method in Example 1:

$$
u(t)=t^{2}
$$

It is also the exact solution and Table 1 presents the results.

Example 2. Consider the following two singularly linear BVPs in [28] Example 1:

$$
\begin{aligned}
& t(1-t) u^{\prime \prime}(t)+(1-t) u^{\prime}(t)+t u(t) \\
& \quad+t(1-t) u^{2}(t)=f(t), \quad 0<t<1,
\end{aligned}
$$

with boundary values $u(0)=0, u(1)=1$, and $f(t)=t(4-$ $\left.4 t+t^{2}+t^{4}-t^{5}\right)$. The exact solution is $u(t)=t^{2}$.

The differential transformation of (35) is

$$
\begin{aligned}
k(k+1) U(k)-k(k-1) U(k)+(k+1) U(k+1) \\
\quad-k U(k)+U(k-1)+B(k-1)-B(k-2) \\
=4 \delta(k-1)-4 \delta(k-2)+\delta(k-3) \\
\quad+\delta(k-5)-\delta(k-6),
\end{aligned}
$$

where $B(k)=U(0) U(k)+U(1) U(k-1)+\cdots+U(k) U(0)$ for $k \geq 2$. Consider

$$
\begin{aligned}
\Longrightarrow & \\
=\frac{1}{(k+1)^{2}}[ & \\
& \\
& \\
& -B(k-1)+4 \delta(k-1)-4 \delta(k-2) \\
& +\delta(k-3)+\delta(k-5)-\delta(k-6)] .
\end{aligned}
$$

For the coefficient of constant and $t$, because $u(0)=0$, let $u(t)=U(1) t+U(2) t^{2}$; substituting in (35), we have

$$
\left(t-t^{2}\right) 2 U(2)+(1-t)[U(1)+2 U(2) t]+t U(1) t .
$$

The coefficient of constant is

$$
U(1)=0 .
$$

The coefficient of $t$ that is $k=1$ is

$$
\begin{gathered}
2 U(2)-U(1)+2 U(2)=4 \\
\Longrightarrow U(2)=1 .
\end{gathered}
$$

As $k=2$ in (37),

$$
U(3)=\frac{4 U(2)-U(1)+B(0)-B(1)-4}{9}=0 .
$$

As $k=3$,

$$
U(4)=\frac{9 U(3)-U(2)+B(1)-B(2)+1}{16}=0 .
$$

For $k=4,5, \ldots$,

$$
U(k+1)=0 .
$$

Then we have the numerical solution of the present method in Example 2 as

$$
u(t)=t^{2}
$$

It is also the exact solution.

Example 3. Consider the following two singularly linear BVPs:

$$
\begin{array}{r}
t(1-t) u^{\prime \prime}(t)+(1-t) u^{\prime}(t)+\left(1+t^{2}-t^{3}\right) u(t)=f(t) \\
0<t<1,
\end{array}
$$

with boundary values $u(0)=1, u(1)=e$, and $f(t)=(2-$ $\left.t^{3}\right) e^{t}$. The exact solution is $u(t)=e^{t}$.

The differential transformation of (45) is

$$
\begin{gathered}
k(k+1) U(k)-k(k-1) U(k)+(k+1) U(k+1)-k U(k) \\
+U(k)+U(k-2)-U(k-3)=\frac{2}{k !}-\frac{1}{(k-3) !},
\end{gathered}
$$


TABLE 2: Comparison of relative errors of the present method for Example 3.

\begin{tabular}{lcc}
\hline$x$ & True solution $u(x)$ & Present $\mathrm{DTM}_{8}$ method \\
\hline 0.01 & 1.0101 & 0 \\
0.08 & 1.0833 & $1.90 E-13$ \\
0.16 & 1.1735 & $7.50 E-12$ \\
0.32 & 1.3771 & $1.00 E-10$ \\
0.48 & 1.6161 & $3.90 E-09$ \\
0.64 & 1.8965 & $5.30 E-08$ \\
0.8 & 2.2255 & $4.00 E-07$ \\
0.96 & 2.6117 & $2.10 E-06$ \\
\hline
\end{tabular}

TABLE 3: Comparison of relative errors of the present method for Example 4.

\begin{tabular}{lccc}
\hline$x$ & True solution $u(x)$ & Geng $[28] U_{5,50}$ & Present $\mathrm{DTM}_{8}$ method \\
\hline 0.08 & 0.0064 & over $1 E-7$ & 0 \\
0.16 & 0.0256 & over $2 E-7$ & $1.9 E-13$ \\
0.24 & 0.0576 & over $2 E-6$ & $7.3 E-12$ \\
0.32 & 0.1024 & over $3 E-6$ & $9.7 E-11$ \\
0.48 & 0.2304 & over $1 E-5$ & $3.7 E-09$ \\
0.64 & 0.4096 & over $1 E-6$ & $4.9 E-08$ \\
0.80 & 0.6400 & over $3 E-6$ & $3.7 E-07$ \\
0.96 & 0.9216 & over $1 E-7$ & $7.0 E-07$ \\
\hline
\end{tabular}

for $k \geq 2$. Consider

$$
\begin{aligned}
\Longrightarrow & U(k+1) \\
=\frac{1}{(k+1)^{2}}[ & \left(k^{2}+1\right) U(k)-U(k-2) \\
& \left.+U(k-3)+\frac{2}{k !}-\frac{1}{(k-3) !}\right] .
\end{aligned}
$$

Because $u(0)=1, U(1)=1$. For $U(1)=1, U(2)=1 / 2$, we have Table 2 .

Example 4. Consider the following two singularly linear BVPs in [28] Example 3:

$$
\begin{aligned}
t^{3}(1-t)^{2} u^{\prime \prime}(t)+5 u^{\prime}(t)+(2+t) u(t)+(1-t)^{3} u^{2}(t) & =f(t) \\
0 & <t<1,
\end{aligned}
$$

with boundary values $u(0)=0, u(1)=1+\sin (1)$, and $f(t)=$ $5(1+\cos (t))-(t-1)^{2} t^{3} \sin (t)+(t+2)(t+\sin (t))-(t-1)^{3}(t+$ $\sin (t))^{2}$. The exact solution is $u(t)=\sin (t)+t$.

Using the same method as the above examples, we have Table 3.

\section{Conclusion}

The modification proposed in this paper has demonstrated that the linear and nonlinear singular BVPs can be handled without difficulty. The computation can obtain more precise approximation. The results show a greater improvement over the He's HPM and RKHSM [28].

How to establish error analysis of this method? Maybe Adomian decomposition method with integrating factor has some beautiful results [29]. In the paper, we only have the convergence of the solution. The underlined theory for two singular points BVPs, however, still remains open and deserves further investigation.

\section{Conflict of Interests}

The authors declare that there is no conflict of interests.

\section{Acknowledgment}

This work was supported by the National Science Council of the Republic of China under Contract 101-2221-E-006-092MY2.

\section{References}

[1] D. Bartolucci and E. Montefusco, "Blow-up analysis, existence and qualitative properties of solutions for the two-dimensional Emden-Fowler equation with singular potential," Mathematical Methods in the Applied Sciences, vol. 30, no. 18, pp. 2309-2327, 2007.

[2] M. Lefranc and J. Mawhin, "Ètude qualitative des solutions de l'équation différentielle d'Emden-Fowler," Académie Royale de Belgique Bulletin de la Classe des Sciences, vol. 55, no. 5, p. 763770, 1969 (French).

[3] C. Guo, C. Zhai, and R. Song, "An existence and uniqueness result for the singular Lane-Emden-Fowler equation," Nonlinear Analysis: Theory, Methods \& Applications, vol. 72, no. 3-4, pp. 1275-1279, 2010.

[4] A. E. Ebaid, "Exact solutions for a class of nonlinear singular two-point boundary value problems: the decomposition method," Zeitschrift fur Naturforschung, vol. 65, no. 3, pp. 145$150,2010$.

[5] J. S. Duan and R. Rach, "A new modification of the Adomian decomposition method for solving boundary value problems for higher order nonlinear differential equations," Applied Mathematics and Computation, vol. 218, no. 8, pp. 4090-4118, 2011.

[6] A. M. Wazwaz, "A new method for solving singular initial value problems in the second-order ordinary differential equations," Applied Mathematics and Computation, vol. 128, no. 1, pp. 45-57, 2002.

[7] M. S. H. Chowdhury and I. Hashim, "Solutions of EmdenFowler equations by homotopy-perturbation method," Nonlinear Analysis: Real World Applications, vol. 10, no. 1, pp. 104-115, 2009.

[8] A. M. Wazwaz, "Adomian decomposition method for a reliable treatment of the Emden-Fowler equation," Applied Mathematics and Computation, vol. 161, no. 2, pp. 543-560, 2005.

[9] A. M. Wazwaz, "A new algorithm for solving differential equations of Lane-Emden type," Applied Mathematics and Computation, vol. 118, no. 2-3, pp. 287-310, 2001. 
[10] P. M. Lima and A. M. Oliveira, "Numerical solution of a singular boundary value problem for a generalized EmdenFowler equation," Applied Numerical Mathematics, vol. 45, no. 4, pp. 389-409, 2003.

[11] S. A. Khuri, "A new approach to Bratu's problem," Applied Mathematics and Computation, vol. 147, no. 1, pp. 131-136, 2004.

[12] P. Y. Tsai and C. K. Chen, "An approximate analytic solution of the nonlinear Riccati differential equation," Journal of the Franklin Institute, vol. 347, no. 10, pp. 1850-1862, 2010.

[13] A. Ebaid, "A new analytical and numerical treatment for singular two-point boundary value problems via the Adomian decomposition method," Journal of Computational and Applied Mathematics, vol. 235, no. 8, pp. 1914-1924, 2011.

[14] G. È. Puhov, "The application of Taylor transforms to the solution of differential equations," Elektronikai Modelirovanie Vyp, vol. 11, no. 128, pp. 18-23, 1976 (Russian).

[15] A. Kurnaz and G. Oturanç, "The differential transform approximation for the system of ordinary differential equations," International Journal of Computer Mathematics, vol. 82, no. 6, pp. 709-719, 2005.

[16] F. Mirzaee, "Differential transform method for solving linear and nonlinear systems of ordinary differential equations," Applied Mathematical Sciences, vol. 5, no. 70, pp. 3465-3472, 2011.

[17] C. K. Chen and S. H. Ho, "Solving partial differential equations by two-dimensional differential transform method," Applied Mathematics and Computation, vol. 106, no. 2-3, pp. 171-179, 1999.

[18] M. J. Jang, C. L. Chen, and Y. C. Liu, "Two-dimensional differential transform for partial differential equations," Applied Mathematics and Computation, vol. 121, no. 2-3, pp. 261-270, 2001.

[19] A. Çetinkaya, O. Kıymaz, and J. Çamlı, "Solutions of nonlinear PDE's of fractional order with generalized differential transform method," International Mathematical Forum, vol. 6, no. 1, pp. 39-47, 2011.

[20] H. Liu and Y. Song, "Differential transform method applied to high index differential-algebraic equations," Applied Mathematics and Computation, vol. 184, no. 2, pp. 748-753, 2007.

[21] F. Ayaz, "Applications of differential transform method to differential-algebraic equations," Applied Mathematics and Computation, vol. 152, no. 3, pp. 649-657, 2004.

[22] L. Zou, Z. Wang, and Z. Zong, "Generalized differential transform method to differential-difference equation," Physics Letters A, vol. 373, no. 45, pp. 4142-4151, 2009.

[23] A. Arikoglu and I. Ozkol, "Solution of differential-difference equations by using differential transform method," Applied Mathematics and Computation, vol. 181, no. 1, pp. 153-162, 2006.

[24] A. Tari and S. Shahmorad, "Differential transform method for the system of two-dimensional nonlinear Volterra integrodifferential equations," Computers and Mathematics with Applications, vol. 61, no. 9, pp. 2621-2629, 2011.

[25] D. Nazari and S. Shahmorad, "Application of the fractional differential transform method to fractional-order integrodifferential equations with nonlocal boundary conditions," Journal of Computational and Applied Mathematics, vol. 234, no. 3, pp. 883-891, 2010.

[26] J. Liu and G. Hou, "Numerical solutions of the space- and timefractional coupled Burgers equations by generalized differential transform method," Applied Mathematics and Computation, vol. 217, no. 16, pp. 7001-7008, 2011.
[27] M. T. Alquran and K. Al-Khaled, "Approximations of SturmLiouville eigenvalues using sinc-Galerkin and differential transform methods," Applications and Applied Mathematics, vol. 5, no. 1, pp. 128-147, 2010.

[28] F. Geng and M. Cui, "Solving singular nonlinear boundary value problems by combining the homotopy perturbation method and reproducing kernel Hilbert space method," International Journal of Computer Mathematics, vol. 87, no. 9, pp. 2024-2031, 2010.

[29] Y. W. Lin, T. T. Lu, and C. K. Chen, "Adomian decomposition method using integrating factor," Communications in Theoretical Physics, vol. 60, no. 2, pp. 159-164, 2013. 


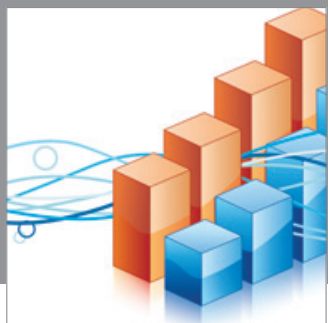

Advances in

Operations Research

mansans

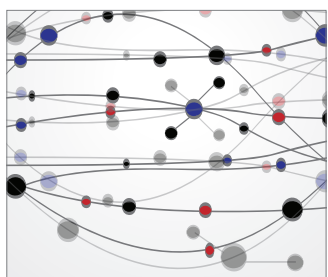

The Scientific World Journal
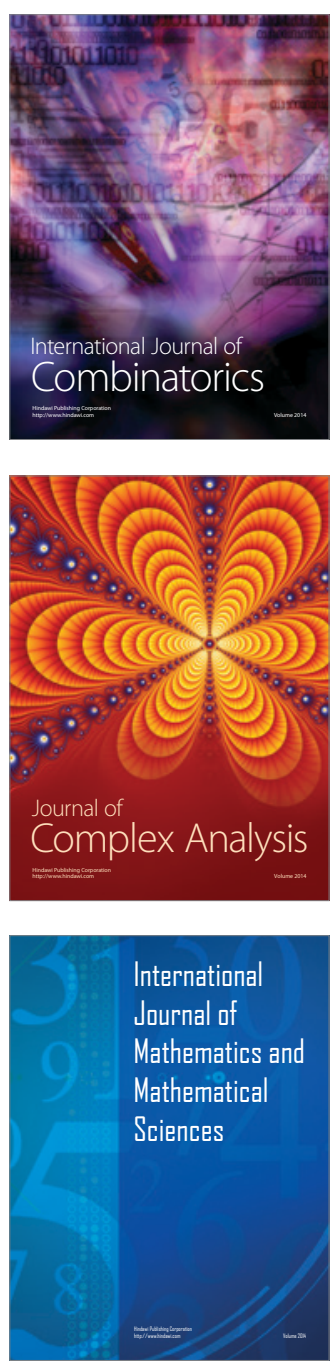
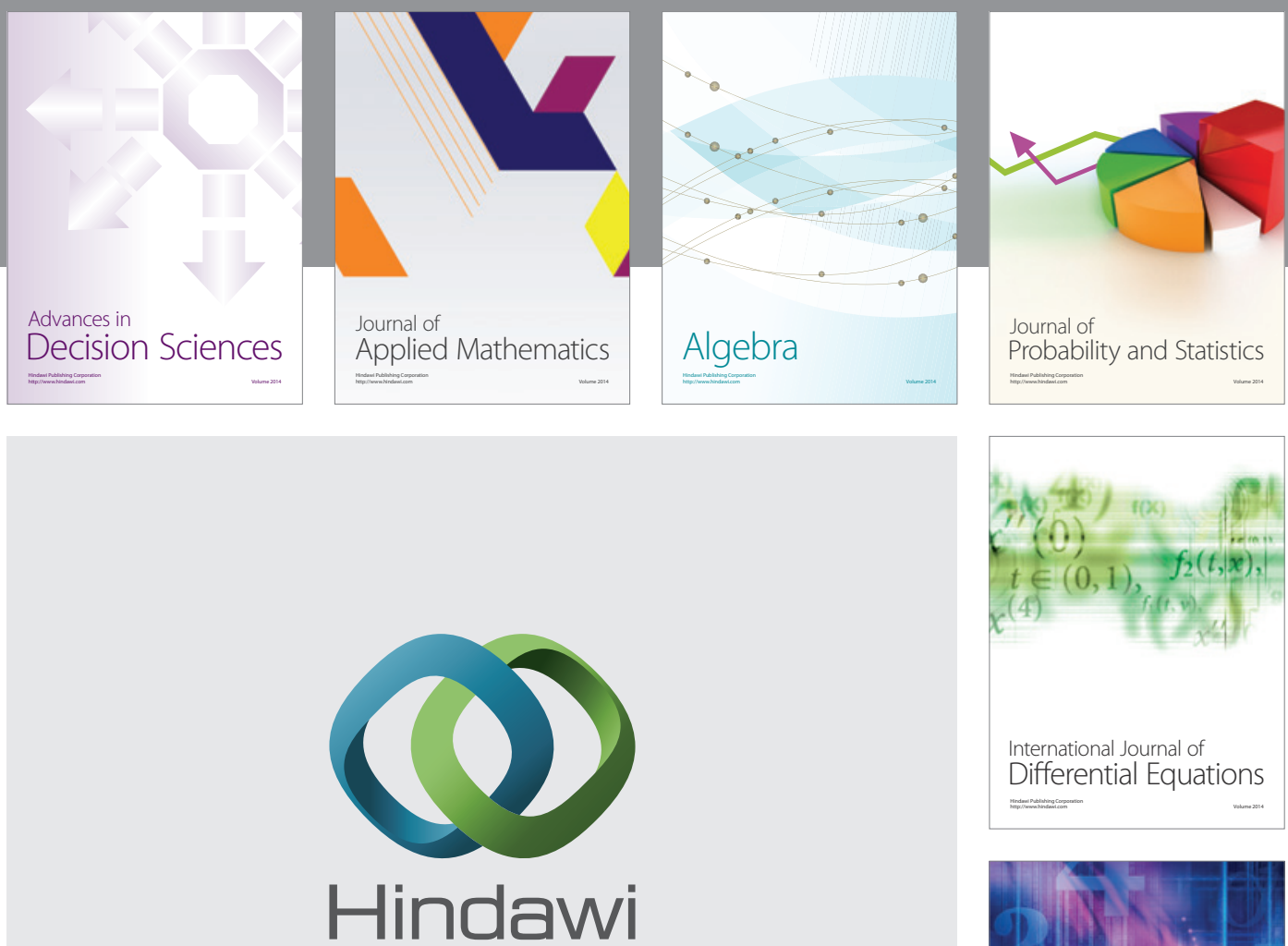

Submit your manuscripts at http://www.hindawi.com
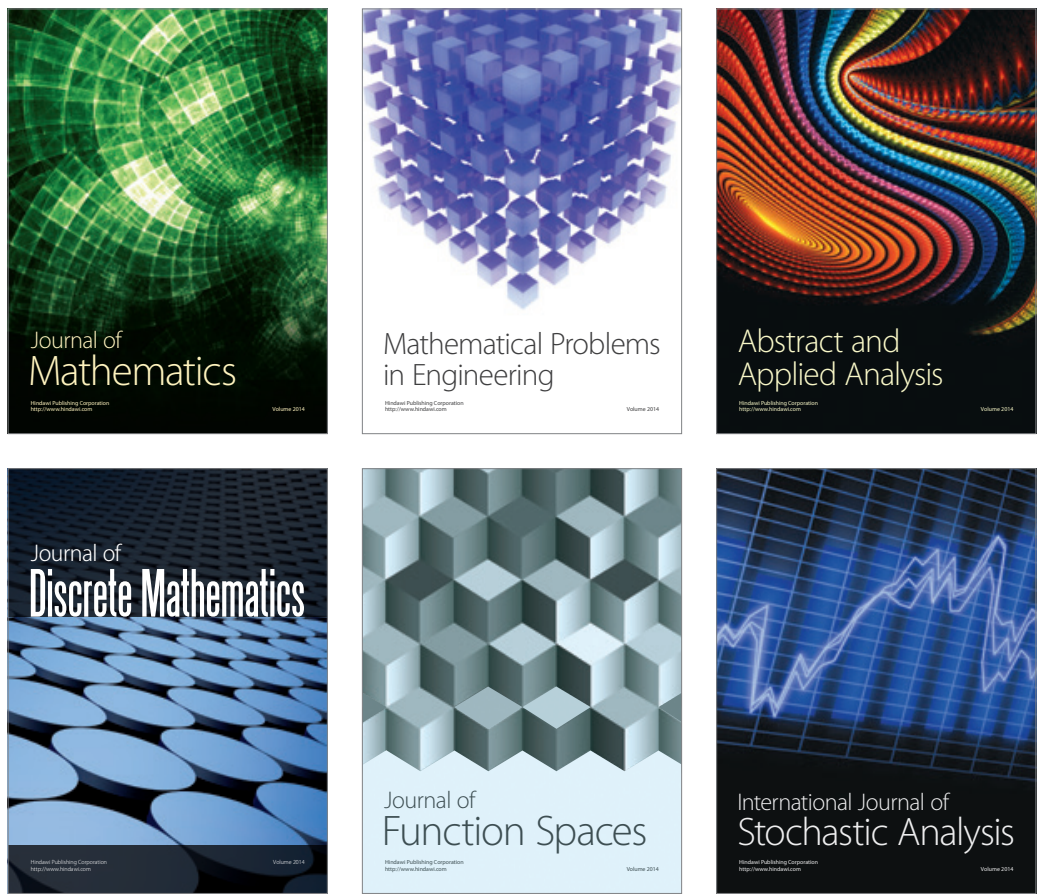

Journal of

Function Spaces

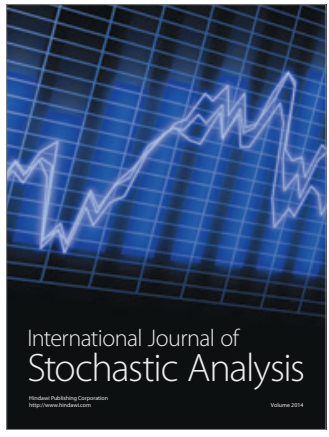

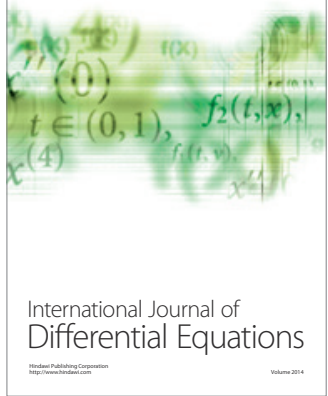
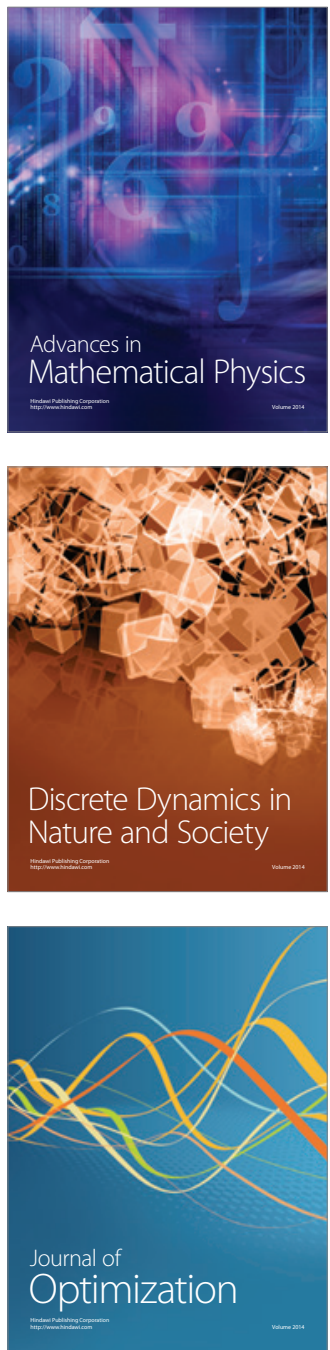